\title{
Active Tuning of the Resonance Frequencies of LC Bandpass Filters for Frequency Domain Multiplexed Readout of TES Detector Arrays
}

\author{
J. van der Kuur ${ }^{1}$ (D) - L. Gottardi' ${ }^{2}$ H. Akamatsu' ${ }^{2}$ - A. C. T. Nieuwenhuizen² \\ R. den Hartog ${ }^{2}$ B. D. Jackson ${ }^{1}$
}

Received: 8 November 2017 / Accepted: 22 August 2018 / Published online: 6 September 2018 (c) The Author(s) 2018

\begin{abstract}
Efficient use of the available frequency space for frequency domain multiplexing is essential for space-based TES detector applications such as the X-IFU instrument on the Athena X-ray telescope. For cross talk reasons, there exists a lower limit on the distance between neighbouring pixels in frequency space, so that accurate control of the resonance frequency helps to use the available frequency space. Independently, from the point of view of efficient sinusoidal bias voltage generation, placing the frequencies on a grid with a fixed spacing helps to avoid the effects of nonlinearity on the detector performance. Inevitable tolerances in the lithographic production of LC bandpass filters and margins in the design of stray inductances limit the relative accuracy of the resonance frequencies to a few times $10^{-3}$. In this paper we will propose a new method which uses the active SQUID readout electronics at room temperature to further fine-tune the resonance frequencies of the LC filters beyond the manufacturing limitations.
\end{abstract}

Keywords TES $\cdot$ FDM $\cdot$ Tuning

\section{Introduction}

Imaging arrays of cryogenic detectors based on transition edge sensors (TES) are nowadays being used for a wide range of ground based applications and are also the baseline detector type for the space-based telescopes Athena and Spica. Especially for space-based applications, power-efficient readout is an essential component. There are

$凶$ J. van der Kuur

j.van.der.kuur@sron.nl

1 SRON Netherlands Institute for Space Research, Landleven 12, 9747 AD Groningen, The Netherlands

2 SRON Netherlands Institute for Space Research, Sorbonnelaan 2, 3584 CA Utrecht, The Netherlands 
several multiplexing schemes in use, which all have in common that the information of a pixel is limited in bandwidth, then mounted on an independent carrier per pixel, and then added to a multiplexed data stream.

Frequency domain multiplexing (FDM) is one of the multiplexing technologies for the readout of TES-based detectors. SRON develops FDM for the readout of the X-IFU instrument on the Athena observatory [1], and for the Safari instrument on the Spica observatory [2]. FDM is implemented by mounting the signals of TES-based pixels on individual carriers by applying sinusoidal bias currents with different frequencies per pixel. A $L C$ bandpass filter per pixel, which is tuned at the frequency of the assigned carrier, ensures that the bandwidth of the signal is confined, and that a single frequency per pixel is applied to generate the bias. Separation in frequency space is essential to keep the signals mathematically independent, so that individual signals can be retrieved from the multiplexed data stream without addition of information from other pixels (i.e., cross talk).

Efficient use of the bandwidth is needed to minimise the power dissipation. As a result, the carrier frequencies should be as closely packed as allowable from cross talk considerations. For the X-IFU instrument on the Athena X-ray observatory, a multiplexing factor of 40 has been chosen as a baseline with carrier frequencies ranging between 1 and $5 \mathrm{MHz}$, with a spacing of $100 \mathrm{kHz}$ [3]. For a more elaborate description of the focal plane assembly, we refer to [4]. In this paper we discuss a method to further reduce the impact of the nonlinearity of the DAC.

\section{Impact of Nonlinearity on FDM}

Nonlinearity in the readout chain or in the carrier generation leads to the generation of higher order harmonics, and therefore spoils in principle strict separation in frequency space. Although monotonic nonlinear transfer functions of the readout chain are mathematically invertible, the inversion would require measurement of the generated harmonics with a sufficient signal-to-noise ratio to avoid significant addition of amplifier noise. As a result, this inversion operation is generally not feasible for high-dynamic-range applications such as X-ray detectors.

In the FDM system under development, the main sources of nonlinearity are the SQUID, the digital-to-analog converters (DACs) for the carrier and carrier nulling signals, and the first-stage low-noise amplifier (LNA) at room temperature.

Nonlinearity in the carrier generation, which leads to spurious signals in the sidebands of the carriers, cannot be inverted as the TES-based pixels are power detectors. As a result, nonlinearity in the amplifier chain and in the bias sources must be kept below a threshold level, which depends on the requirements of the application.

The amplitude of the harmonics generated by a nonlinear transformation is a strong function of the amplitude of transformed signal. This implies that for a given nonlinear transfer function of a component in a system, the impact on the nonlinearity can be minimised by minimising the amplitude of the transferred signals. This approach is applied to counter the effects on nonlinearity of the SQUIDs and of the LNA, by using baseband feedback $[5,6]$ to null the stationary carriers and the superimposed timedependent carrier modulation in the forward path of the baseband feedback loops. 
This method cannot be applied for the generation of the carriers and the carrier nulling signals, as the full amplitudes are unavoidable to generate the required bias power for the detectors, and to null the resulting carriers at the input of the SQUID. Note that because the nonlinearity of the SQUIDs and LNAs are stronger than the nonlinearity of the DACs, it is advantageous to minimise the SQUID signals as opposed to the DAC signals.

\section{Carrier Frequencies on a Grid}

To further minimise the impact of the nonlinearity present in the DACs we make use of the fact that the amplitude-modulated signal from the TES is only a small fraction of the stationary bias current. We can write the modulated signals with index $i$ as $I_{i}(t)=\left(I_{0, i}+\Delta I_{i}(t)\right) \sin \omega_{i} t$, with $I_{0, i}$ the amplitude of the stationary bias current, and $\Delta I_{i}(t)$ the time-dependent part of the amplitude that is produced by the noise and the photon responses, and $\omega_{i}$ the angular frequency of the sinusoidal TES bias current of the TES with index $i$. For the X-IFU instrument, the energy dependence of the X-ray optics [7] causes that for the vast majority of the absorbed photons we find that $\Delta I_{i}(t) \lesssim 0.1 I_{0, i}$. As a result, the harmonic products with the largest amplitudes will be produced by the stationary part of the carriers.

In order to efficiently use the available multiplex bandwidth, the carrier frequencies are placed on a regular grid. The distance in frequency space between adjacent carriers is set by cross talk requirements which dictate a minimum ratio between the frequency distance between neighbours, and the bandwidth per pixel. The minimum bandwidth per pixel, in turn, is set by the speed of the pixel and the electrothermal stability requirements. Because of inevitable tolerances on the resonance frequency of the $L C$ filters, the carrier frequencies cannot be on an exact regular grid. The best result on the accuracy of the resonance frequencies reported so far shows a spread of $2.3 \mathrm{kHz}$ $(=0.7 \%$ ) around the designed resonance frequencies can be achieved in the frequency range of $1-3 \mathrm{MHz}$ [8].

The harmonics created by nonlinearity are always integer multiples of the frequency differences between the carriers. As a result, the harmonics have a high probability of ending up in the side bands of pixels at the higher carrier frequencies. The impact of a harmonic in the side band carrying energy information of a detected photon depends on the instantaneous phase of the harmonic at the moment a photon is absorbed, and on the frequency difference between the harmonic and the carrier frequency of the pixel it is affecting. As the exact location of the harmonics depends on the statistical spread on the designed resonance frequencies of the $L C$ bandpass filters, one must use a worst-case estimate when specifying the maximum allowable amplitudes of the harmonics. For the X-IFU instrument this turns out to impose stringent requirements on the spurious free dynamic range (SFDR) of the DACs.

When we choose the angular carrier frequencies $\omega_{i}$ such that they are exact integer multiples of each other, i.e., $\omega_{i}=\omega_{0}+n \cdot \omega_{\Delta}$, with $\omega_{0}=2 \pi f_{0}$ the lowest angular bias frequency with frequency $f_{0}, n \geq 0$ a positive integer, and $\omega_{\Delta}$ the angular frequency distance between two adjacent carriers, the harmonics with the largest amplitudes will be stationary, and with frequencies sitting on the same equispaced grid as the 
angular bias frequencies $\omega_{i}$. This, in turn, implies that the largest effect of the DAC nonlinearity will be an amplitude offset of the carrier only, instead of a harmonic in the side band. Therefore, the impact of the nonlinearity will be much smaller when the carrier frequencies are on an equispaced grid.

The angular resonance frequency $\omega_{0}$ of a $L C$ bandpass filter with inductance $L$ and capacitance $C$, in series with a (TES) resistor $R_{0}$ in the limit of $R_{0} \ll \sqrt{L / C}$, depends of both the values of $L$ and $C$, as $\omega_{0}=1 / \sqrt{L C}$. Resonance frequency tuning can thus be obtained by changing either $L$ or $C$. In the same limit, the angular electrical bandwidth $B_{\text {pix }}$ of the circuit is set by $R_{0}$ and $L$ only, i.e., $B_{\text {pix }} \equiv R_{0} / L$. Therefore, active frequency tuning is preferably implemented by tuning the capacitors, as the bandwidth of the bias circuit is constrained by the electrothermal stability requirements of the TES.

\section{Operating Principle and Implementation of Active Frequency Tuning}

A circuit element in an electrical network can be seen as a device which measures the current, and creates a voltage proportional to the observed current. In the case of a resistor, inductor, or capacitor the resulting voltage is proportional to the current directly, to its derivative, or to the integral of the observed current, respectively. This implies that if the instantaneous current in the circuit is known, and if the output voltage of the active bias source in the circuit can be adjusted based on the observed current, an active impedance can be created in series with the active bias source. This active impedance adds to the impedance of the passive components in the circuit, so that the net impedance can be changed in this manner.

In a FDM readout system with baseband feedback [9], one has access to both the measured current through and the applied voltage to the TES. As a result, by creating a feedback path between the measured current and applied voltage per pixel, a virtual resistor, capacitor, or inductor can be projected in the TES bias circuit, with both positive and negative signs. The resulting net impedance of the TES bias circuit consists then of the impedance of the physical $L C$ bandpass filter in series with the actively created impedances.

A schematic diagram of the feedback scheme in the frequency domain for a single pixel in the small signal approximation is shown in Fig. 1. To simplify the expressions, they have been evaluated around the angular bias carrier frequency $\omega_{\mathrm{c}}$. We therefore define $\Delta \omega \equiv \omega-\omega_{\mathrm{c}}$ with $\Delta \omega \ll \omega_{0}$, and $\Delta \omega_{L C} \equiv \omega_{\mathrm{c}}-\omega_{L C}$ the shift induced by active frequency tuning with respect to the angular resonance frequency $\omega_{L C}$ of the $L C$ filter.

The forward path contains the conventional readout chain consisting of the impedance of the TES $Z_{\text {tes }}$ in series with the $L C$ bandpass filter, and the transfer $A_{\text {sq }}$ of the SQUID chain which is limited by a single pole at an angular frequency of $1 / \tau$ which is in first order approximation equal to the gain-bandwidth product of the baseband feedback loop. The feedback path is designed such that its impedance equals the impedance of the $L C$ filter at $\omega_{\mathrm{c}}$, so that the net transfer of the circuit becomes $I_{m}=V_{0, c} /\left(Z_{\text {tes }}+2 j L \Delta \omega\right)$ in the limit where the gain-bandwidth product 


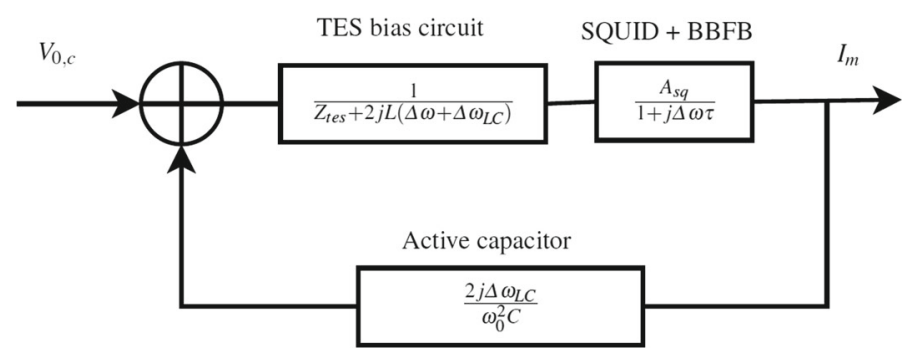

Fig. 1 Schematic diagram of the feedback scheme in the frequency domain for a single pixel, in the small signal approximation, and evaluated around the angular bias carrier frequency $\omega_{\mathrm{c}}$ for simplicity. We therefore define $\Delta \omega \equiv \omega-\omega_{\mathrm{c}}$ with $\Delta \omega \ll \omega_{0}$, and $\Delta \omega_{L C} \equiv \omega_{\mathrm{c}}-\omega_{L C}$ the shift induced by active frequency tuning with respect to the angular resonance frequency $\omega_{L C}$ of the $L C$ filter. The forward path contains the conventional readout chain consisting of the impedance of the TES $Z_{\text {tes }}$ in series with the $L C$ bandpass filter, and the transfer $A_{\mathrm{sq}}$ of the SQUID chain which is limited by a single pole at an angular frequency of $1 / \tau$ which is in first order approximation equal to the gain-bandwidth product of the baseband feedback loop. The feedback path is designed such that its impedance equals the impedance of the $L C$ filter at $\omega_{\mathrm{c}}$, so that the net transfer of the circuit becomes $I_{m}=V_{0, c} /\left(Z_{\text {tes }}+2 j L \Delta \omega\right)$ in the limit where, the $1 / \tau \gg B_{\text {pix }}$ and $A_{\mathrm{sq}}=1$

of the baseband feedback loop is significantly larger than the electrical bandwidth of the pixel, i.e., $1 / \tau \gg B_{\text {pix }}$. We therefore can conclude that the net angular resonance frequency of the circuit has changed from $\omega_{L C}$ to $\omega_{\mathrm{c}}$, as desired. The implementation of the active capacitor in the firmware of the digital readout electronics of FDM requires an integrator and a multiplier per pixel, though more implementation forms are conceivable. The functionality of the circuit has been demonstrated in simulations. Experimental demonstrations are underway.

The voltage drop across the active capacitor must be created by the digital-to-analog converter (DAC) which supplies the bias voltage to the TESs and therefore increases the dynamic range requirements of the DAC by a factor $\sqrt{1+\left(2 \Delta \omega_{L C} L / R_{0}\right)^{2}}$ with $R_{0}$ the set-point resistance of the TES. With the shown standard deviation of $2.4 \mathrm{kHz}$ on $\omega_{L C} /(2 \pi)$, and a bandwidth per pixel of $2-3 \mathrm{kHz}$, we expect this effect to be of the order of $\sqrt{2}$. On the other hand the requirements for the SFDR become less stringent because the spurious signals can be kept outside the detector information band of $\sim 1 \mathrm{kHz}$, where their effect is attenuated by the standard matched filter. In an optimised system, this can reduce the SFDR requirements by possibly an order of magnitude, so we expect still a net relieve of the SFDR requirements. The dynamic range requirement for the SQUID also increases by a factor $\sqrt{1+\left(2 \Delta \omega_{L C} L / R_{0}\right)^{2}}$ when active frequency tuning is applied. This results from the fact that the measured current, which is contaminated with SQUID noise, is used to create the active capacitor.

\section{Summary and Conclusions}

Nonlinear transfer functions in the readout chain of TES-based detectors leads to creation of harmonics, with cross talk as a result. The impact of SQUID and amplifier nonlinearity is minimised by baseband feedback, which minimises the signal ampli- 
tude in these nonlinear elements. The resulting nulling signals have the full amplitudes, and are created by DACs. To minimise the impact of the DAC nonlinearity, it is attractive to put the carrier frequencies on an equispaced grid. Production tolerances on the analog cryogenic $L C$ components together with the need to use the bandwidth efficiently, inhibits the use of an equispaced grid.

We propose that an active feedback between the measured current per pixel and the applied voltage per pixel can be used to effectively change the resonance frequency of the TES bias circuit. In this way the goal to use an equispaced grid can be achieved at the cost of slight extra dynamic range requirements on the SQUID. Simulations of the transfers of the TES bias circuit together with baseband feedback and the active frequency tuning technique in the Z-domain in Simulink ${ }^{\circledR}$ have shown the functionality of the circuit.

Acknowledgements The research leading to these results has received funding from the European Unions Horizon 2020 Programme under the AHEAD project (Grant agreement n. 654215).

Open Access This article is distributed under the terms of the Creative Commons Attribution 4.0 International License (http://creativecommons.org/licenses/by/4.0/), which permits unrestricted use, distribution, and reproduction in any medium, provided you give appropriate credit to the original author(s) and the source, provide a link to the Creative Commons license, and indicate if changes were made.

\section{References}

1. D. Barret, T.L. Trong, J.-W. den Herder, L. Piro, X. Barcons, J. Huovelin, R. Kelley, J.M. Mas-Hesse, K. Mitsuda, S. Paltani, G. Rauw, A. Rożanska, J. Wilms, M. Barbera, E. Bozzo, M.T. Ceballos, I. Charles, A. Decourchelle, R. den Hartog, J.-M. Duval, F. Fiore, F. Gatti, A. Goldwurm, B. Jackson, P. Jonker, C. Kilbourne, C. Macculi, M. Mendez, S. Molendi, P. Orleanski, F. Pajot, E. Pointecouteau, F. Porter, G.W. Pratt, D. Prêle, L. Ravera, E. Renotte, J. Schaye, K. Shinozaki, L. Valenziano, J. Vink, N. Webb, N. Yamasaki, F. Delcelier-Douchin, M. Le Du, J.-M. Mesnager, A. Pradines, G. Branduardi-Raymont, M. Dadina, A. Finoguenov, Y. Fukazawa, A. Janiuk, J. Miller, Y. Nazé, F. Nicastro, S. Sciortino, J.M. Torrejon, H. Geoffray, I. Hernandez, L. Luno, P. Peille, J. André, C. Daniel, C. Etcheverry, E. Gloaguen, J. Hassin, G. Hervet, I. Maussang, J. Moueza, A. Paillet, B. Vella, G. Campos Garrido, J.-C. Damery, C. Panem, J. Panh, S. Bandler, J.-M. Biffi, K. Boyce, A. Clénet, M. DiPirro, P. Jamotton, S. Lotti, D. Schwander, S. Smith, B.-J. van Leeuwen, H. van Weers, T. Brand, B. Cobo, T. Dauser, J. de Plaa, and E. Cucchetti. The Athena X-ray Integral Field Unit (X-IFU). ArXiv arXiv:1608.08105, August (2016)

2. B. Sibthorpe, F. Helmich, P. Roelfsema, H. Kaneda, H. Shibai, The SPICA mission, vol 75-76 of EAS Publications Series, M1 - Conference Proceedings (2016), pp. 411-417

3. J. van der Kuur, L.G. Gottardi, H. Akamatsu, B.J. van Leeuwen, R. den Hartog, D. Haas, M. Kiviranta, and B.J. Jackson, Optimising the multiplex factor of the frequency domain multiplexed readout of the tes-based microcalorimeter imaging array for the $\mathrm{x}$-ifu instrument on the athena $\mathrm{x}$-ray observatory, in Proceedings of SPIE, vol. 9905 (2016), pp .99055R-99055R-9 http://arxiv.org/abs/1611.05268

4. B.D. Jackson, H. van Weers, J. van der Kuur, R. den Hartog, H. Akamatsu, A. Argan, S.R. Bandler, M. Barbera, D. Barret, M.P. Bruijn, J.A. Chervenak, J. Dercksen, F. Gatti, L. Gottardi, D. Haas, J.-W. den Herder, C.A. Kilbourne, M. Kiviranta, T. Lam-Trong, B.-J. van Leeuwen, C. Macculi, L. Piro, S.J. Smith, The focal plane assembly for the athena x-ray integral field unit instrument, in Proceedings of SPIE, vol. 9905 (2016), pp .99052I-99052I-8

5. M. Kiviranta, H. Seppä, J. van der Kuur, P. de Korte, SQUID-based readout schemes for microcalorimeter arrays. In F.S. Porter, D. McCammon, M. Galeazzi, and C.K. Stahle, (eds.) in AIP Conference Proceedings LTD-9, vol. 605 AIP (2002), pp. 295-300

6. Roland den Hartog, D. Boersma, M. Bruijn, B. Dirks, L. Gottardi, H. Hoevers, R. Hou, M. Kiviranta, P. de Korte, J. van der Kuur, B.J. van Leeuwen, A. Nieuwenhuizen, M. Popescu, Baseband feedback 
for frequency domain multiplexed readout of tes x ray detectors, in AIP Conference Proceedings, vol. 1185, no. (1) (2009). pp. 261-264

7. J.W. den Herder, D. Bagnali, S. Bandler, M. Barbera, X. Barcons, D. Barret, P. Bastia, M. Bisotti, K. Boyce, C. Cara, M. Ceballos, L. Corcione, B. Cobo, L. Colasanti, J. de Plaa, M. DiPirro, W.B. Doriese, Y. Ezoe, R. Fujimoto, F. Gatti, L. Gottardi, P. Guttridge, R. den Hartog, I. Hepburn, R. Kelley, K. Irwin, Y. Ishisaki, C. Kilbourne, P.A.J. de Korte, J. van der Kuur, S. Lotti, C. Macculi, K. Mitsuda, T. Mineo, L. Natalucci, T. Ohashi, M. Page, S. Paltani, E. Perinati, L. Piro, C. Pigot, F.S. Porter, G. Rauw, L. Ravera, E. Renotte, J.-L. Sauvageot, C. Schmid, S. Sciortino, P. Shirron, Y. Takei, G. Torrioli, M. Tsujimoto, L. Valenziano, D. Willingale, C. de Vries, H. van Weers, J. Wilms, N.Y. Yamasaki, The X-ray microcalorimeter spectrometer onboard athena. vol. 8443 (2012), pp. 8443-8443-12

8. M.P. Bruijn, L. Gottardi, R.H. den Hartog, J. van der Kuur, A.J. van der Linden, B.D. Jackson, Tailoring the high-q lc filter arrays for readout of kilo-pixel tes arrays in the spica-safari instrument. J. Low Temp Phys 176(3-4), 421-425 (2014)

9. L. Ravera, C. Cara, M.T. Ceballos, X. Barcons, D. Barret, R. Cldassou, A. Clnet, B. Cobo, E. Doumayrou, R.H. den Hartog, B.-J. van Leeuwen, D. van Loon, J.M. Mas-Hesse, C. Pigot, E. Pointecouteau, The dre: the digital readout electronics for athena x-ifu, in Proceedings of SPIE, vol. 9144 (2014), pp. 91445T-91445T-8 